\title{
Estabilidade de sistemas não lineares com saltos Markovianos sob condição de ortogonalidade entre controle e termos não lineares
}

\author{
Alessandro N. Vargas* Sérgio M. Filho*, \\ Cristiano M. Agulhari* Marcio A. F. Montezuma* \\ Eduardo F. Costa ${ }^{* *}$ \\ * Universidade Tecnológica Federal do Paraná, UTFPR, \\ Av. Alberto Carazzai 1640, 86300-000 Cornelio Procópio-PR, Brazil \\ ** Universidade de São Paulo, USP, \\ Depto. Matemática Aplicada e Estatística, C.P. 668, 13560-970, São \\ Carlos-SP, Brazil
}

\begin{abstract}
This paper presents conditions to assure the second moment stability for a class of nonlinear Markov jump systems. The control and nonlinear terms form orthogonal vectors, a novel condition in the context of Markov jump systems. The second moment stability is verified through linear matrix inequalities, and a numerical evaluation illustrates the main result.

Resumo: Este artigo apresenta condições para assegurar a estabilidade de segundo momento para uma classe de sistemas com saltos Markovianos não lineares. Vetores dos termos que dependem do controle e função não linear formam produto ortogonal, sendo esta a condição inédita deste trabalho. A estabilidade de segundo momento é verificada através de desigualdades matriciais lineares, e um exemplo numérico ilustra o principal resultado do artigo.
\end{abstract}

Keywords: Stochastic systems; Stochastic control; stability; nonlinear systems.

Palavras-chaves: Sistemas estocásticos; Controle estocástico; estabilidade; sistemas não lineares.

\section{INTRODUÇÃO}

Nos últimos anos, os sistemas sujeitos a saltos Markovianos têm recebido atenção especial pois são úteis para modelar sistemas dinâmicos sujeitos a variações bruscas aleatórias. Exemplos de aplicações de sistemas com saltos Markovianos podem ser encontrados em robótica Vargas et al. (2013b), economia Costa and de Oliveira (2012); Oliveira et al. (2009), redes Zhang et al. (2013); Li et al. (2015a) e motores elétricos Oliveira et al. (2014); Vargas et al. (2013a); Yin et al. (2014).

Existem diversos resultados para controle de sistemas não lineares com saltos Markovianos como Shi and Li (2015); Yin and Zhu (2010). Por exemplo, podemos mencionar controle para lidar com não linearidades em sensores em Rao et al. (2018), quantização Li et al. (2015b), realimentação de saída em Wu et al. (2014), redes baseadas em eventos em Zha et al. (2017), e estratégias através de modos deslizantes em Li et al. (2014, 2016); Liu et al. (2011).

Alguns dos diversos trabalhos focados em condições para estabilidade podem ser encontrados na monografia Costa et al. (2013) e nos artigos Khasminskii et al. (2007); Long and Zhong (2017); Vargas et al. (2017); Zhang and Boukas (2009); Zhao and Gupta (2015). Aqui contribuímos para através de resultados inovadores que garantem a estabilidade em segundo momento para o sistema não linear controlado aqui abordado.

Em termos formais, considere um espaço de probabilidade filtrado e fixado $(\Omega, \mathcal{F}, P)$ governando a evolução do seguinte sistema estocástico.

$$
\begin{aligned}
\mathrm{d} x(t)= & \left(A_{\theta(t)} x(t)+\left[\begin{array}{c}
\mathbf{0}_{q} \\
B_{\theta(t)}
\end{array}\right] u(t)\right) \mathrm{d} t \\
& +\left[\begin{array}{c}
g_{\theta(t)}(x(t)) \\
\mathbf{0}_{n-q}
\end{array}\right] \mathrm{d} t+H_{\theta(t)} \mathrm{d} w(t), \quad \forall t \geq 0,
\end{aligned}
$$

com $x(0)=x_{0} \in \mathbb{R}^{n}$, onde $x(t) \in \mathbb{R}^{n}, u(t) \in \mathbb{R}^{m}$, e $w(t) \in \mathbb{R}^{r}$ representam os estados do sistema, entrada de controle, e movimento Browniano, respectivamente. O processo $\{\theta(t)\}$ denota uma cadeia de Makov em tempo contínuo e irredutível assumindo valores no conjunto $\mathcal{I}=$ $\{1, \ldots, N\}$.

As funções $g_{i}: \mathbb{R}^{n} \mapsto \mathbb{R}^{q}, i=1, \ldots, N$, são mensuráveis e precisamente conhecidas. As matrizes $A_{\theta(t)}=A_{i}, B_{\theta(t)}=$ $B_{i}$, e $H_{\theta(t)}=H_{i}$ são dadas para $\theta(t)=i \in \mathcal{I}$.

Note em (1) que os vetores considerados no termo de controle e no termo não linear são ortogonais, i.e.,

$$
u(t)^{\prime}\left[\begin{array}{ll}
\mathbf{0}_{q}^{\prime} & B_{\theta(t)}^{\prime}
\end{array}\right]^{\prime}\left[\begin{array}{r}
g_{\theta(t)}(x(t)) \\
\mathbf{0}_{n-\text { DOL }} \mathbf{1 0 . 1 7 6}
\end{array}\right]=0 .
$$


Esta ortogonalidade possui uma motivação prática-Seção 4 apresenta dados em tempo real de um equipamento automotivo que requer esta ortogonalidade na prática.

Esta ortogonalidade, ainda, nos permite explorar a estrutura particular do sistema (1) para desenvolver o principal resultado usando condições mais fracas que as existentes na literatura -vide Observação 7. De acordo com o conhecimento dos autores, este artigo é o primeiro a considerar ortogonalidade entre termos não lineares e de controle no contexto de sistemas com saltos Markovianos.

A lei de controle em (1) está na forma linear de realimentação de estados

$$
u(t)=K_{\theta(t)} x(t), \quad \forall t \geq 0 .
$$

Definição 1. ((Arnold, 1974, Defn. 11.3.1, p. 188)(Vargas et al., 2017, Defn. 1.1, p. 333)). O sistema não linear com saltos Markovianos em (1) é estável em segundo momento se existe alguma constante $c=c\left(x_{0}\right)$ tal que

$$
\mathrm{E}\left[\|x(t)\|^{2}\right] \leq c, \quad \forall t \geq 0
$$

A contribuição principal deste artigo é mostrar condições que tornam o sistema com saltos Markovianos em (1) sujeito a (2) estável em segundo momento. As condições se dão em termos de desigualdades lineares matriciais, passíveis de computação. O principal resultado deste artigo pode ser interpretado como a primeira tentativa de caracterizar condições de estabilidade para sistemas não lineares com saltos Markovianos considerando ortogonalidade entre termos não lineares e termos de controle.

\subsection{Motivação adicional em sistemas não lineares com saltos Markovianos}

A representação em (1) é útil para modelagem de uma classe mais ampla de sistemas com saltos Markovianos. Por exemplo, para o sistema não linear com saltos Markovianos na forma

$$
\begin{gathered}
\mathrm{d} x(t)=\left(A_{\theta(t)} x(t)+\left[\begin{array}{c}
g_{\theta(t), 1}(x(t)) \\
\vdots \\
g_{\theta(t), n}(x(t))
\end{array}\right]\right) \mathrm{d} t \\
+\left[\begin{array}{c}
\mathbf{0}_{q} \\
B_{\theta(t)}
\end{array}\right](\psi(x(t))+\gamma(x(t)) u(t)) \mathrm{d} t+H_{\theta(t)} \mathrm{d} w(t), \forall t \geq 0,
\end{gathered}
$$

$x(0)=x_{0} \in \mathbb{R}^{n}$, onde $\gamma(x)$ e $\psi(x)$ representam funções não lineares, e $\gamma(x)$ é não singular, nos inspiramos no método de linearização da realimentação de estados, que é uma técnica útil que converte uma equação de estados não lineares em uma equação linear (Khalil, 2002, p. 506), para definir o controlador $u(t)$ como

$$
\begin{aligned}
& u(t)=\gamma(x(t))^{-1}[-\psi(x(t)) \\
& \left.+K_{\theta(t)} x(t)-B_{\theta(t)}^{+}\left[\begin{array}{c}
g_{\theta(t), q+1}(x(t)) \\
\vdots \\
g_{\theta(t), n}(x(t))
\end{array}\right]\right],
\end{aligned}
$$

onde $B_{\theta(t)}^{+}$representa a inversa de Moore-Penrose de $B_{\theta(t)}$, e $B_{\theta(t)}$ possui colunas linearmente independentes.

Comentário 2. Os sistemas (1) e (3) são equivalente so- para calcular $K_{\theta(t)}$ de modo que (1) se torna estável em segundo momento - isto representa a contribuição principal deste artigo.

\section{NOTAÇÕES E RESULTADOS AUXILIARES}

O espaço Euclidiano n-dimensional é denotado por $\mathbb{R}^{n}$, e sua norma correspondente é representada por $\|$.$\| . O$ operador traço é designado por $\operatorname{tr}\{$.$\} . A notação da matriz$ identidade em $\mathbb{R}^{n \times n}$ é $I_{n}$. O símbolo $\mathbb{1}_{\mathcal{C}}$ se refere à medida de Dirac de $\mathcal{C}$, i.e., se a condição $\mathcal{C}$ é verdadeira, então $\mathbb{1}_{\mathcal{C}}$ é igual a 1 , caso contrário é igual a 0 . Dadas duas matrizes $U \in \mathbb{R}^{n \times n}$ e $V \in \mathbb{R}^{m \times m}$, a matriz bloco diagonal formada por $V$ e $U$ é representada por $\operatorname{diag}(V, U)$. No caso $\operatorname{de} \operatorname{diag}\left(V_{1}, \ldots V_{N}\right)$, definimos $\operatorname{diag}\left(V_{i}\right)_{\{i=1, \ldots, N\}}$.

Quando desejamos explicitar o $\ell$-ésimo elemento do vetor $v \in \mathbb{R}^{n}$, usamos a notação $v_{[\ell]}$.

A notação $\mathbb{S}^{n}$ será utilizada para se referir ao conjunto das matrizes simétricas definidas positivas de dimensão $n$.

O processo homogêneo $\theta=\left\{\left(\theta(t), \mathcal{F}_{t}\right), t \in \mathbb{R}^{+}\right\}$assume valores no conjunto $\mathcal{I}=\{1, \ldots, N\}$.

Assumimos ainda que

$$
\operatorname{Pr}(\theta(t+h)=j \mid \theta(t)=i)=\left\{\begin{array}{l}
\pi_{i j} h+o(h), \quad i \neq j, \\
1+\pi_{i j} h+o(h), \quad i=j,
\end{array}\right.
$$

onde $\left[\left(\pi_{i j}\right)\right]$ é a matriz de taxa de transição estacionária de $\{\theta\} \operatorname{com} 0 \leq \pi_{i j}, i \neq j$, e $0 \leq \pi_{i}:=-\pi_{i i}=\sum_{\{j: j \neq i\}} \pi_{i j}$ para todo $i \in \mathcal{I}, o(h)$ se refere a um infinitesimal de ordem maior que $h$, i.e., $\lim _{h \rightarrow 0} \frac{o(h)}{h}=0$. Assumimos que $x(0), w(t)$, e $\theta(t)$ são variáveis aleatórias mutuamente independentes para cada $t \geq 0$.

Lema 3. (Lema da Projeção (Pipeleers et al., 2009, p.511)). Dada uma matriz simétrica $-Z \in \mathbb{S}^{m}$, e duas matrizes arbitrárias $U$ e $V$ com coluna de dimensão $m$, então existe uma matriz não estruturada $X$ que satisfaz $U^{\prime} X V+V^{\prime} X^{\prime} U+Z<0$, se, e somente se, as seguintes desigualdades de projeção em relação a $X$ são satisfeitas: $N_{U}^{\prime} Z N_{U}<0$ e $N_{V}^{\prime} Z N_{V}<0$, onde $N_{U}$ e $N_{V}$ são matrizes arbitrárias cujas colunas formam uma base para os espaços nulos de $U$ e $V$, respectivamente.

\subsection{Resultados básicos para sistemas lineares autônomos com saltos Markovianos}

Considere o sistema linear autônomo com saltos Markovianos

$$
\dot{z}(t)=A_{\theta(t)} z(t), \quad t \geq 0, \quad \theta(0)=\theta_{0}, \quad z(0)=z_{0} \in \mathbb{R}^{n} .
$$

Definição 4. ((Costa et al., 2013, Defn. 3.2 p.36)(Fragoso and Costa, 2005, Defn. 3.2, p.1173)). Dizemos que o sistema em (5) é estável na média quadrática se $\mathrm{E}\left[\|z(t)\|^{2}\right] \rightarrow$ 0 quando $t \rightarrow+\infty$ para todo $z_{0} \in \mathbb{R}^{n}$.

O próximo resultado será útil na prova do principal resultado deste artigo.

Lema 5. O sistema em (5) é estável na média quadrática se existem matrizes $P_{i}$ 's em $\mathbb{S}^{n}$ e $X_{i}$ em $\mathbb{R}^{n \times n}$ tais que (para cada $i=1, \ldots, N)$,

DOI: $10.17648 /$ sbai-2019-111172 


$$
\left[\begin{array}{ccc}
A_{i} X_{i}+X_{i}^{\prime} A_{i}^{\prime} & P_{i}+A_{i} X_{i}-X_{i}^{\prime} & R_{i} \\
P_{i}+X_{i}^{\prime} A_{i}^{\prime}-X_{i} & -X_{i}-X_{i}^{\prime} & -X_{i} \\
R_{i} & -X_{i}^{\prime} & A_{i} X_{i}+X_{i}^{\prime} A_{i}^{\prime}-R_{i}
\end{array}\right]<0,
$$

onde $R_{i}=\sum_{j=1}^{N} \pi_{j i} P_{j}$.

Prova. Faça $Z \in \mathbb{R}^{3 n \times 3 n}$ de modo que

$$
Z=\left[\begin{array}{ccc}
0 & P_{i} & R_{i} \\
P_{i} & 0 & 0 \\
R_{i} & 0 & -R_{i}
\end{array}\right]
$$

Defina $N_{U}^{\prime}=\left[\begin{array}{lll}I & A_{i} & I\end{array}\right]$ and $N_{V}^{\prime}=\left[\begin{array}{lll}-I & I & 0\end{array}\right]$. Segue que

$$
\begin{aligned}
N_{U}^{\prime} Z N_{U} & =\left[\begin{array}{lll}
I & A_{i} & I
\end{array}\right]\left[\begin{array}{ccc}
0 & P_{i} & R_{i} \\
P_{i} & 0 & 0 \\
R_{i} & 0 & -R_{i}
\end{array}\right]\left[\begin{array}{c}
I \\
A_{i}^{\prime} \\
I
\end{array}\right] \\
& =A_{i} P_{i}+P_{i} A_{i}^{\prime}+R_{i},
\end{aligned}
$$

e que

$$
N_{V}^{\prime} Z N_{V}=\left[\begin{array}{lll}
-I & I & 0
\end{array}\right]\left[\begin{array}{ccc}
0 & P_{i} & R_{i} \\
P_{i} & 0 & 0 \\
R_{i} & 0 & -R_{i}
\end{array}\right]\left[\begin{array}{c}
-I \\
I \\
0
\end{array}\right]=-2 P_{i}<0 .
$$

Agora, escolha $U$ e $V$ tais que as colunas de $N_{U}$ e $N_{V}$ formam uma base para os espaçoes nulos de $U$ e $V$, respectivamente; deste modo, faça $U=\left[\begin{array}{ccc}A_{i}^{\prime} & -I & 0 \\ 0 & -I & A_{i}^{\prime}\end{array}\right]$ tal que

$$
U N_{U}=\left[\begin{array}{ccc}
A_{i}^{\prime} & -I & 0 \\
0 & -I & A_{i}^{\prime}
\end{array}\right]\left[\begin{array}{c}
I \\
A_{i}^{\prime} \\
I
\end{array}\right]=0
$$

and $V=\left[\begin{array}{lll}I & I & 0 \\ 0 & 0 & I\end{array}\right]$ para obter

$$
V N_{V}=\left[\begin{array}{lll}
I & I & 0 \\
0 & 0 & I
\end{array}\right]\left[\begin{array}{c}
-I \\
I \\
0
\end{array}\right]=0
$$

Considere $X_{11, i}=X_{22, i}=X_{i}$, e $X_{12, i}=X_{21, i}=\mathbf{0}$. Se a próxima desigualdade for satisfeita

$$
\begin{gathered}
{\left[\begin{array}{ccc}
0 & P_{i} & R_{i} \\
P_{i} & 0 & 0 \\
R_{i} & 0 & -R_{i}
\end{array}\right]+\left[\begin{array}{cc}
A_{i} & 0 \\
-I & -I \\
0 & A_{i}
\end{array}\right]\left[\begin{array}{cc}
X_{11, i} & X_{12, i} \\
X_{21, i} & X_{22, i}
\end{array}\right]\left[\begin{array}{ccc}
I & I & 0 \\
0 & 0 & I
\end{array}\right]} \\
+\left[\begin{array}{ll}
I & 0 \\
I & 0 \\
0 & I
\end{array}\right]\left[\begin{array}{cc}
X_{11, i}^{\prime} & X_{21, i}^{\prime} \\
X_{12, i}^{\prime} & X_{22, i}^{\prime}
\end{array}\right]\left[\begin{array}{ccc}
A_{i}^{\prime} & -I & 0 \\
0 & -I & A_{i}^{\prime}
\end{array}\right] \\
=\left[\begin{array}{ccc}
A_{i} X_{i}+X_{i}^{\prime} A_{i}^{\prime} & P_{i}+A_{i} X_{i}-X_{i}^{\prime} & R_{i} \\
P_{i}+X_{i}^{\prime} A_{i}^{\prime}-X_{i} & -X_{i}-X_{i}^{\prime} & -X_{i} \\
R_{i} & -X_{i}^{\prime} & A_{i} X_{i}+X_{i}^{\prime} A_{i}^{\prime}-R_{i}
\end{array}\right] \\
\quad \begin{array}{c}
<,
\end{array}
\end{gathered}
$$

então o Lema 3 implica que $N_{U}^{\prime} Z N_{U}<0$, o que resulta de (8) que $A_{i} P_{i}+P_{i} A_{i}^{\prime}+R_{i}<0$. Esta desigualdade garante que os sitema em (5) é estável na média quadrática (Costa et al., 2013, Thm. 3.21 p.48), (Fragoso and Costa, 2005, Thm. 4.19, p.1179).

\section{RESULTADO PRINCIPAL}

Escrevamos a equação diferencial estocástica não linear (1)

$$
\begin{aligned}
\mathrm{d}\left[\begin{array}{l}
x_{1}(t) \\
x_{2}(t)
\end{array}\right] & =\left(\left[\begin{array}{ll}
A_{11, \theta(t)} & A_{12, \theta(t)} \\
A_{21, \theta(t)} & A_{22, \theta(t)}
\end{array}\right]\left[\begin{array}{l}
x_{1}(t) \\
x_{2}(t)
\end{array}\right]\right. \\
+ & {\left.\left[\begin{array}{c}
\mathbf{0}_{q} \\
B_{\theta(t)}
\end{array}\right] u(t)\right) \mathrm{d} t+\left[\begin{array}{c}
g_{\theta(t), 1}(x(t)) \\
\vdots \\
g_{\theta(t), q}(x(t)) \\
\mathbf{0}_{n-q}
\end{array}\right] \mathrm{d} t } \\
& +H_{\theta(t)} \mathrm{d} w(t),
\end{aligned}
$$

com $x_{1}(t) \in \mathbb{R}^{q}$ e $x_{2}(t) \in \mathbb{R}^{n-q}$. A motivação para escrever o sistema (10) em tal forma particionada será esclarecida na sequência.

A próxima hipótese é inspirada na condição de crescimento linear (veja (Mao et al., 2007, Assump. 3.1), (Li et al., 2017, Assump. 1)).

Hipótese 6 . Existem duas constantes positivas $\alpha_{1}$ e $\alpha_{2}$ tais que

$$
\begin{aligned}
& x_{[\ell]} g_{i, \ell}(x) \leq \alpha_{2}+\alpha_{1} x_{[\ell]}^{2}, \quad \forall x \in \mathbb{R}^{n}, \\
& i=1, \ldots, N, \quad \ell=1, \ldots, q .
\end{aligned}
$$

Comentário \%. Os resultados disponíveis na literatura necessitam que as desigualdades em (11) sejam satisfeitas para cada $\ell=1, \ldots, n$ ((Mao et al., 2007, Assump. 3.1), (Li et al., 2017, Assump. 1), (Yin and Zhu, 2010, Thm. 2.1, p. 30)); entretanto, nossa abordagem requer estas desigualdades somente para $\ell=1, \ldots, q$ (se lembre que $q<n)$. A necessidade de menos desigualdades é uma clara vantagem da nossa abordagem que deriva da condição de ortogonalidade entre os termos de controle e não lineares, como será discutido adiante.

Denote $p_{i}(t):=\operatorname{Pr}(\theta(t)=i)$ para cada $i \in \mathcal{I}$. Considere a matriz semi definida positiva $V_{i}(t)$ em $\mathbb{S}^{m}$, solução da equação diferencial matricial ( $\alpha_{1}$ e $\alpha_{2}$ abaixo representam as constantes da Hipótese 6)

$$
\begin{gathered}
\dot{V}_{i}(t)=V_{i}(t)\left(\tilde{A}_{i}+\alpha_{1} I\right)^{\prime}+\left(\tilde{A}_{i}+\alpha_{1} I\right) V_{i}(t) \\
+\sum_{j=1}^{N} \pi_{j i} V_{j}(t)+H_{i} H_{i}^{\prime} p_{i}(t)+2 \alpha_{2} I, \\
\quad i=1, \ldots, N, \quad \forall t \geq t_{0}, \quad V_{i}\left(t_{0}\right) \in \mathbb{S}^{m},
\end{gathered}
$$

onde as matrizes de malha fechada $\tilde{A}_{i}$ 's satisfazem

$$
\tilde{A}_{i}:=\left[\begin{array}{cc}
A_{11, i} & A_{12, i} \\
A_{21, i}+B_{i} K_{i, 1} & A_{22, i}+B_{i} K_{i, 2}
\end{array}\right], \quad i=1, \ldots, N .
$$

Hipótese 6 é importante na prova do próximo resultado.

Lema 8. Defina a matriz de segundo momento

$$
\mathcal{X}_{i}(t)=\mathrm{E}\left[x(t) x(t)^{\prime} \mathbb{1}_{\theta(t)=i}\right], \quad i=1, \ldots, N, \quad \forall t \geq 0,
$$

onde $x(t) \in \mathbb{R}^{n}$ denota a solução de (1). Então existe algum $t_{0} \geq 0$ tal que

$$
\operatorname{tr}\left\{\mathcal{X}_{i}(t)\right\} \leq \operatorname{tr}\left\{V_{i}(t)\right\}, \quad i=1, \ldots, N, \quad \forall t \geq t_{0},
$$

onde $V_{i}(t)$ satisfaz $(12) \operatorname{com} V_{i}\left(t_{0}\right)=\mathcal{X}_{i}\left(t_{0}\right)$.

A prova do Lema 8 segue de uma adaptação do (Vargas et al., 2017, Thm. 3.1) e por isso é omitida.

Comentário 9. Se $V(t)$ é uniformemente limitado, então o segundo momento $\mathrm{E}\left[\|x(t)\|^{2}\right]$ é também uniforme-

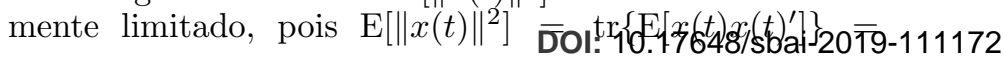




$$
\begin{aligned}
& {\left[\begin{array}{ccc}
M_{i}+M_{i}^{\prime}+\alpha_{1}\left(X_{i}+X_{i}^{\prime}\right) & P_{i}+M_{i}+\alpha_{1} X_{i}-X_{i}^{\prime} & R_{i} \\
P_{i}+M_{i}^{\prime}+X_{i}^{\prime} \alpha_{1}-X_{i} & -X_{i}-X_{i}^{\prime} & -X_{i} \\
R_{i} & -X_{i}^{\prime} & M_{i}+M_{i}^{\prime}+\alpha_{1}\left(X_{i}+X_{i}^{\prime}\right)-R_{i}
\end{array}\right]<0,} \\
& M_{i}=\left[\begin{array}{cc}
A_{11, i} X_{1, i} & A_{12, i} X_{2, i} \\
A_{21, i} X_{1, i}+B_{i} Y_{1, i} & A_{22, i} X_{2, i}+B_{i} Y_{2, i}
\end{array}\right], \quad i=1, \ldots, N .
\end{aligned}
$$

$\sum_{i=1}^{N} \operatorname{tr}\left\{\mathcal{X}_{i}(t)\right\}$, o que é limitada superiormente por $\sum_{i=1}^{N} \operatorname{tr}\left\{\mathcal{V}_{i}(t)\right\}$ devido a (16).

Agora estamos aptos a apresentar o resultado principal deste artigo.

Teorema 10. Suponha que existam matrizes não estrutu$\operatorname{radas} Y_{1, i}, Y_{2, i}, X_{1, i}, X_{2, i}$, com $X_{i}=\operatorname{diag}\left(X_{1, i}, X_{2, i}\right)$, e matrizes definidas positivas $P_{i}$ 's em $\mathbb{S}^{n}$ tais que as desigualdades em (14) sejam satisfeitas. Então o sistema linear autônomo com saltos Markovianos

$$
\dot{z}(t)=\left(\tilde{A}_{\theta(t)}+\alpha_{1} I\right) z(t), \quad z(0)=z_{0} \in \mathbb{R}^{n},
$$

é estável na média quadrática, e o ganho de controle $K_{i}$, $i=1, \ldots, N$, requeridos em (2), e também em (13), é dado por

$$
K_{i}=\left[\begin{array}{lll}
Y_{1, i} X_{1, i}^{-1} & Y_{2, i} X_{2, i}^{-1}
\end{array}\right] .
$$

Prova. Multiplique a matriz $\tilde{A}_{i}$ em (13) por $X_{i}=$ $\operatorname{diag}\left(X_{1, i}, X_{2, i}\right)$ para obter

$$
M_{i}=\tilde{A}_{i} X_{i}=\left[\begin{array}{cc}
A_{11, i} X_{1, i} & A_{12, i} X_{2, i} \\
A_{21, i} X_{1, i}+B_{i} Y_{1, i} & A_{22, i} X_{2, i}+B_{i} Y_{2, i}
\end{array}\right] .
$$

Fazendo $Y_{1, i}=K_{1, i} X_{1, i}$ e $Y_{2, i}=K_{2, i} X_{2, i}$, obtemos

$$
\tilde{A}_{i} X_{i}=\left[\begin{array}{cc}
A_{11, i} X_{1, i} & A_{12, i} X_{2, i} \\
\left(A_{21, i}+B_{i} K_{1, i}\right) X_{1, i} & \left(A_{22, i}+B_{i} K_{2, i}\right) X_{2, i}
\end{array}\right] .
$$

Substituindo (18) em (14) resulta

$$
\left[\begin{array}{ccc}
T_{i} & P_{i}+\left(\tilde{A}_{i}+\alpha_{1} I\right) X_{i}-X_{i}^{\prime} & R_{i} \\
P_{i}+X_{i}^{\prime}\left(\tilde{A}_{i}^{\prime}+\alpha_{1} I\right)-X_{i} & -X_{i}-X_{i}^{\prime} & -X_{i} \\
R_{i} & -X_{i}^{\prime} & T_{i}-R_{i}
\end{array}\right]<0
$$

com

$$
T_{i}:=\left(\tilde{A}_{i}+\alpha_{1} I\right) X_{i}+X_{i}^{\prime}\left(\tilde{A}_{i}^{\prime}+\alpha_{1} I\right) .
$$

O resultado então segue de (19) e do Lema 5 .

Proposição 11. ((Fragoso and Costa, 2005, Thm 5.6), (Costa et al., 2013, Thm. 3.25, p. 52),(Vargas et al., 2017, Prop. 3.1, p. 335)). Se o sistema em (17) é estável na média quadrática, então o limite $\lim _{t \rightarrow \infty} V(t)$ em (12) existe e não depende da condição inicial $V\left(t_{0}\right) \in \mathbb{R}^{n \times n}$.

A existência de $\lim _{t \rightarrow \infty} V(t)$ pela Proposição 11 garante que $V(t)$ é uniformemente limitado; este fato, Lema 8 , e Comentário 9 nos permite concluir que (3) é estável em segundo momento. Esta afirmação é resumida no próximo corolário.

Corolário 12. Sob as condições do Teorema 10, o sistema não linear com saltos Markovianos em (3) (ou em (10)) é estável em segundo momento.

\section{SIMULAÇÃO: CONTROLE PARA UMA VÁLVULA BORBOLETA AUTOMOTIVA}

Esta seção apresenta uma simulação de um modelo de uma válvula borboleta automotiva Vargas et al. (2016).

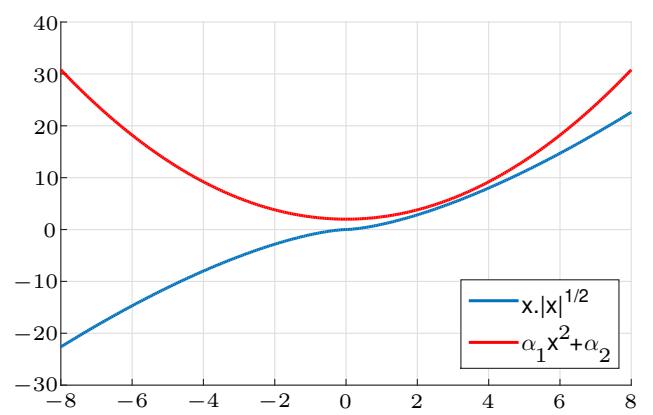

Figura 1. Duas curvas ilustram que Hipótese 6 vale quando $\alpha_{1}=0.45$ e $\alpha_{2}=2$.

Aqui se supõe que falhas abruptas afetam o driver da válvula, sob dois modos de operação. O primeiro modo, i.e, $\theta(t)=1$, representa a válvula trabalhando sob perfeitas condições. O segundo modo, i.e, $\theta(t)=2$, representa a falha, que impõe o trabalho (esforço) a tão somente $25 \%$ de sua tensão elétrica original. Aqui impomos também o termo não linear $\left|x_{[3]}(t)\right|^{\frac{1}{2}}$ no sistema a seguir para fins de ilustração somente.

$$
\begin{gathered}
\mathrm{d}\left[\begin{array}{l}
x_{[1]}(t) \\
x_{[2]}(t) \\
x_{[3]}(t)
\end{array}\right]=\left(\left[\begin{array}{ccc}
0 & a_{12} & 0 \\
a_{21} & a_{22} & a_{23} \\
0 & a_{32} & a_{33}
\end{array}\right]\left[\begin{array}{l}
x_{[1]}(t) \\
x_{[2]}(t) \\
x_{[3]}(t)
\end{array}\right]+\left[\begin{array}{c}
0 \\
0 \\
b_{\theta(t)}
\end{array}\right] u(t)\right. \\
\left.+\left[\begin{array}{c}
\left|x_{[3]}(t)\right|^{\frac{1}{2}} \\
0 \\
0
\end{array}\right]\right) \mathrm{d} t+\mathrm{d} w(t) .
\end{gathered}
$$

\subsection{Controle}

Os parâmetros $a_{12}, \ldots, a_{33}$, estão listados na Tab. 4.1, sendo que $b(1)=-0.58$ e $b(2)=-0.145$.

Pode-se perceber que a Hipótese 6 vale quando $\alpha_{1}=0.45$ e $\alpha_{2}=2$ porque $x|x|^{1 / 2} \leq \alpha_{2}+\alpha_{1} x^{2}$ para todo $x \in \mathbb{R}$, veja Fig. 1.

Avaliamos as LMIs em (14) com matriz estocástica $\pi_{11}=$ $\pi_{22}=-5 ; \pi_{12}=\pi_{21}=5$. Um ganho factível tomado desde (14) é

$$
K_{i}=\left[\begin{array}{lll}
-0.5-0.005-0.5
\end{array}\right], \quad i=1,2 .
$$

Disto pode-se afirmar que o sistema não linear com saltos Markovianos em (20) é estável em segundo momento sob controle $u(t)=K_{\theta(t)} x(t)$.

$\begin{array}{cc}\text { Parâmetro } & \text { Valor } \\ a_{12} & 0.8106 \\ a_{21} & -58.08 \\ a_{22} & -21.11 \\ a_{23} & -7019 \\ a_{33} & -64.34\end{array}$

Tabela 1. Parâmetros em (20). 


\section{CONCLUSÃO}

Este artigo apresentou condições LMI (desigualdades matriciais lineares) que caracterizam a estabilidade de sistemas estocásticos não lineares. Considera-se um sistema de depende de saltos Markovianos não linear, e assume-se como hipótese de trabalho que o vetor de controle e vetor não linear são ortogonais. Esta hipótese mostra-se útil ao permitir simplificar condições de teste de estabilidade, que de maneira geral estão atreladas à existência de funções de Lyapunov estocástica (ver Yin and Zhu (2010)); aqui não há necessidade de garantir funções de Lyapunov devido a condição de ortogonalidade, de cujo resultado pode ser testado via LMI.

\section{REFERENCIAS}

Arnold, L. (1974). Stochastic differential equations: Theory and applications. Wiley-Interscience, Hoboken, NJ, USA.

Costa, O.L.V. and de Oliveira, A. (2012). Optimal meanvariance control for discrete-time linear systems with Markovian jumps and multiplicative noises. Automatica, 48(2), 304-315.

Costa, O.L.V., Fragoso, M.D., and Todorov, M.G. (2013). Continuous-Time Markov Jump Linear Systems. Series: Probability and Its Applications. Springer-Verlag, New York.

Fragoso, M. and Costa, O. (2005). A unified approach for stochastic and mean square stability of continuous-time linear systems with Markovian jumping parameters and additive disturbances. SIAM J. Control Optim., 44(4), $1165-1191$.

Khalil, H.K. (2002). Nonlinear systems, 3rd. Prentice Hall, Inc, Upper Saddle River, NJ, USA.

Khasminskii, R.Z., Zhu, C., and Yin, G. (2007). Stability of regime-switching diffusions. Stochastic Processes and their Applications, 117(8), 1037-1051.

Li, F., Shi, P., Wu, L., Basin, M.V., and Lim, C. (2015a). Quantized control design for cognitive radio networks modeled as nonlinear semi-Markovian jump systems. IEEE Transactions on Industrial Electronics, 62(4), 2330-2340.

Li, F., Shi, P., Wu, L., Basin, M.V., and Lim, C.C. (2015b). Quantized control design for cognitive radio networks modeled as nonlinear semi-Markovian jump systems. IEEE Transactions on Industrial Electronics, 62(4), 2330-2340.

Li, H., Gao, H., Shi, P., and Zhao, X. (2014). Faulttolerant control of Markovian jump stochastic systems via the augmented sliding mode observer approach. Automatica, 50(7), 1825-1834.

Li, H., Shi, P., and Yao, D. (2017). Adaptive sliding-mode control of Markov jump nonlinear systems with actuator faults. IEEE Transactions on Automatic Control, 62(4), 1933-1939.

Li, H., Shi, P., Yao, D., and Wu, L. (2016). Observer-based adaptive sliding mode control for nonlinear Markovian jump systems. Automatica, 64, 133-142.

Liu, M., Shi, P., Zhang, L., and Zhao, X. (2011). Faulttolerant control for nonlinear Markovian jump systems via proportional and derivative sliding mode observer technique. IEEE Transactions on Circuits and Systems I: Regular Papers, 58(11), 2755-2764.
Long, S. and Zhong, S. (2017). Improved results for stochastic stabilization of a class of discrete-time singular Markovian jump systems with time-varying delay. Nonlinear Analysis: Hybrid Systems, 23, 11-26.

Mao, X., Yin, G.G., and Yuan, C. (2007). Stabilization and destabilization of hybrid systems of stochastic differential equations. Automatica, 43(2), 264-273.

Oliveira, R.C.L.F., Vargas, A.N., do Val, J.B.R., and Peres, P.L.D. (2009). Robust stability, H2 analysis and stabilisation of discrete-time Markov jump linear systems with uncertain probability matrix. Internat. J. Control, 82(3), 470-481.

Oliveira, R.C.L.F., Vargas, A.N., do Val, J.B.R., and Peres, P.L.D. (2014). Mode-independent H2-control of a DC motor modeled as a Markov jump linear system. IEEE Trans. Control Systems Tech., 22(5), 1915-1919.

Pipeleers, G., Demeulenaere, B., Swevers, J., and Vandenberghe, L. (2009). Extended LMI characterizations for stability and performance of linear systems. Systems $\mathbb{G}$ Control Letters, 58(7), 510-518.

Rao, H.X., Xu, Y., Liu, C., and Li, J.Y. (2018). Finite-time control for periodic systems with Markov jump sensor nonlinearities and random input gains. Journal of the Franklin Institute, 355(10), 4097-4110.

Shi, P. and Li, F. (2015). A survey on Markovian jump systems: Modeling and design. International Journal of Control, Automation and Systems, 13(1), 1-16.

Vargas, A.N., Costa, E.F., and do Val, J.B.R. (2013a). On the control of Markov jump linear systems with no mode observation: application to a DC motor device. Int. J. Robust Nonlinear Control, 23(10), 1136-115.

Vargas, A.N., Furloni, W., and do Val, J.B.R. (2013b). Second moment constraints and the control problem of Markov jump linear systems. Numer. Linear Algebra Appl., 20(2), 357-368.

Vargas, A.N., Acho, L., Pujol, G., Costa, E.F., Ishihara, J.Y., and do Val, J.B.R. (2016). Output feedback of Markov jump linear systems with no mode observation: An automotive throttle application. International Journal of Robust and Nonlinear Control, 26(9), 1980-1993.

Vargas, A.N., Pujol, G., and Acho, L. (2017). Stability of Markov jump systems with quadratic terms and its application to RLC circuits. Journal of the Franklin Institute, 354(1), 332-344.

Wu, L., Su, X., and Shi, P. (2014). Output feedback control of Markovian jump repeated scalar nonlinear systems. IEEE Transactions on Automatic Control, 59(1), 199204.

Yin, G.G. and Zhu, C. (2010). Hybrid Switching Diffusions: Properties and Applications. Series: Stochastic Modelling and Applied Probability. Springer, New York, USA.

Yin, Y., Shi, P., Liu, F., and Lim, C.C. (2014). Robust control for nonhomogeneous Markov jump processes: An application to DC motor device. Journal of the Franklin Institute, 351(6), 3322-3338.

Zha, L., an Fang, J., Li, X., and Liu, J. (2017). Eventtriggered output feedback $H_{\infty}$ control for networked Markovian jump systems with quantizations. Nonlinear Analysis: Hybrid Systems, 24, 146-158.

Zhang, L., Gao, H., and Kaynak, O. (2013). Networkinduced constraints in networked control systems - a survey. IEEE Trans. Industrial Informatics, 9(1), 403- 
416.

Zhang, L. and Boukas, E.K. (2009). Stability and stabilization of Markovian jump linear systems with partly unknown transition probabilities. Automatica, 42(2), 463-468.

Zhao, Y. and Gupta, V. (2015). Feedback stabilization of Bernoulli jump nonlinear systems: A passivity-based approach. IEEE Transactions on Automatic Control, $60(8), 2254-2259$. 